\title{
Tailoring of structure formation and phase composition in reactively sputtered zirconium oxide films using nitrogen as an additional reactive gas
}

\author{
D. Severin, Kostas Sarakinos, O. Kappertz, A. Pflug and M. Wuttig
}

\section{Post Print}

N.B.: When citing this work, cite the original article.

Original Publication:

D. Severin, Kostas Sarakinos, O. Kappertz, A. Pflug and M. Wuttig, Tailoring of structure formation and phase composition in reactively sputtered zirconium oxide films using nitrogen as an additional reactive gas, 2008, Journal of Applied Physics, (103), 8, 083306.

http://dx.doi.org/10.1063/1.2903492

Copyright: American Institute of Physics (AIP) http://www.aip.org/

Postprint available at: Linköping University Electronic Press http://urn.kb.se/resolve?urn=urn:nbn:se:liu:diva-71484 


\title{
Tailoring of structure formation and phase composition in reactively sputtered zirconium oxide films using nitrogen as an additional reactive gas
}

\author{
D. Severin, ${ }^{1}$ K. Sarakinos, ${ }^{1}$ O. Kappertz, ${ }^{2}$ A. Pflug, ${ }^{3}$ and M. Wuttig ${ }^{1, a)}$ \\ ${ }^{1}$ Institute of Physics (IA), RWTH Aachen University, 52056 Aachen, Germany \\ ${ }^{2}$ Solid State Electronics Division, Uppsala Universitet, 75120 Uppsala, Sweden \\ ${ }^{3}$ Fraunhofer IST, 38108 Braunschweig, Germany
}

(Received 5 August 2007; accepted 30 January 2008; published online 28 April 2008)

\begin{abstract}
The structure of $\mathrm{ZrO}_{2}$ films has been controlled during reactive sputtering in an argon/oxygen atmosphere by adding an amount of nitrogen gas to the process. Depending on the deposition conditions, amorphous, cubic, or monoclinic films have been obtained without any additional substrate heating. The resulting film structure is explained in terms of the control of fast negative oxygen ions generated at the target surface and accelerated toward the growing film. Furthermore, the nitrogen addition leads to a pronounced stabilization of the plasma discharge and fewer arcing events, while the incorporation of nitrogen atoms in the growing film is very small. (C) 2008 American Institute of Physics. [DOI: 10.1063/1.2903492]
\end{abstract}

\section{INTRODUCTION}

Zirconium oxide $\left(\mathrm{ZrO}_{2}\right)$ is a well known functional coating material with superior properties such as high melting point $\left(2860^{\circ} \mathrm{C}\right)$, high refractive index, and excellent thermal stability. Due to these features, $\mathrm{ZrO}_{2}$ films are employed in numerous applications. These include ionic conductors in the Y-stabilized cubic structure, protective coatings, as well as interference filters. Depending on the temperature, $\mathrm{ZrO}_{2}$ exists in three different stable phases. In particular, (i) the monoclinic phase is obtained between room temperature and $1170{ }^{\circ} \mathrm{C}$, (ii) the tetragonal phase from 1170 to $2370{ }^{\circ} \mathrm{C}$, and (iii) finally the cubic one from 2370 to $2680^{\circ} \mathrm{C}$. A number of deposition techniques including physical and chemical vapor depositions have been employed to deposit zirconium oxide films. In all cases, the crystal structure and the phase composition of $\mathrm{ZrO}_{2}$ films were found to be strongly affected by the energy input during growth with ion beam assisted deposition (IBAD), i.e., substrate temperature and bombardment by energetic species. ${ }^{1}$

Among the various techniques, reactive magnetron sputtering from a metallic target ${ }^{2}$ is widely used since it combines relatively high deposition rates, good homogeneity, and process flexibility. Generally, the reactive sputter process with oxygen is characterized by three different distinct process modes depending on the oxygen gas flow as described by the model proposed by Berg and Nyberg. ${ }^{3}$ At relatively low $\mathrm{O}_{2}$ flow, the process is in the metallic target mode, and is characterized by a high deposition rate, a low coverage (poisoning) of the target surface by the corresponding compound (oxide) and the formation of understoichiometric films. At relatively high oxygen flow, the compound sputter mode is reached. Basic features of this mode are the high oxygen coverage of the target surface and formation of stoichiometric oxide films. The deposition rate in this mode is very low due to the low erosion rate of the oxide compared to that of the corresponding metallic target. Between these regimes,

${ }^{a)}$ Electronic mail: wuttig@physik.rwth-aachen.de. the process is highly unstable in the so-called transition zone. Characteristic for this zone is the abrupt poisoning of the target surface with the oxide when the oxygen gas flow is increased above a threshold value. Furthermore, in this regime, very often a pronounced hysteresis behavior is observed and in many cases no stable working points can be achieved without active process control.

Another important feature of the reactive sputtering of several oxides including $\mathrm{ZrO}_{2}$ is the formation of negatively charged oxygen ions on the poisoned fraction of the target surface. $^{4-11}$ These ions are accelerated within the cathode dark space by the negative target voltage toward the substrate and impinge on the growing film with energies higher than those from the other plasma species. It is therefore evident that their energy depends on the magnitude of the target potential, while the number of energetic ions is determined by the target coverage with the oxide layer. It has been reported in a number of studies that these ions have a strong influence on the structure formation of metal oxides. ${ }^{9,12,13}$ It is therefore essential to control the fraction of the target that is covered with the oxide in order to tailor the bombardment of the growing film by high energy oxygen ions.

In a previous study, we have demonstrated that the addition of nitrogen gas enables the control of the target poisoning by oxygen and the undesired hysteresis zone during reactive sputtering of oxides. ${ }^{14}$ In particular, the abrupt poisoning of the target surface by the oxide with increasing oxygen gas flow (when no nitrogen is added to the process) was altered to a gradual transition from the metallic to the compound oxide mode when nitrogen was present in the gas atmosphere. The higher erosion rate of the nitride compared to the oxide compound was the reason for the vanishing hysteresis. In general, a higher erosion rate of the compound leads to a smoother transition. The addition of nitrogen yields a coexistence of a nitride and an oxide compound on the target surface. Furthermore, the smoothening effect was found to be more pronounced when the amount of nitrogen gas fed into the oxide process was increased. On the other 
hand, the nitrogen incorporation in the growing film was very small at sufficient oxygen partial pressure because the nitrogen on the film surface could be replaced by the more reactive oxygen. This replacement also took place at the target surface. However, the nitride compound is more likely sputtered away before being oxidized. Therefore, the effective target coverage with nitrogen could be much higher than the corresponding nitrogen incorporation in the film.

In this report, we show that the control of the target poisoning with oxygen by the addition of nitrogen gas into the discharge process enables us to tailor the structure formation of $\mathrm{ZrO}_{2}$ due to the variation of the bombardment with fast oxygen ions generated at the target surface. At the same, time the nitrogen incorporation in the film is very small for a sufficient oxygen flow. Hence, a powerful approach to modify the structure of oxide films without a need for additional substrate heating is described.

\section{EXPERIMENT}

Zirconium oxide films with thicknesses between 200 and $450 \mathrm{~nm}$ were deposited on microscope slides employing reactive dc magnetron sputtering at room temperature. Initially, the sputter chamber was evacuated to a base pressure of $\sim 10^{-4} \mathrm{~Pa}$ by a turbo molecular pump with an effective pumping speed of approximately $100 \mathrm{l} / \mathrm{s}$. The films were sputtered from a metallic $\mathrm{Zr}$ target with a purity of $99.9 \%$ and a diameter of $75 \mathrm{~mm}$. Depositions were carried out at a constant cathode current of $400 \mathrm{~mA}$ in a mixed $\mathrm{Ar}-\mathrm{N}_{2}-\mathrm{O}_{2}$ ambient. In particular, three sets of films were deposited at constant $\mathrm{N}_{2}$ flows of $0,0.75$, and $1.5 \mathrm{sccm}$ (sccm denotes cubic centimeter per minute at STP), respectively. For each set, the oxygen flow was increased, while the Ar flow was systematically varied in order to sustain a constant working pressure of $0.8 \mathrm{~Pa}$. The target to substrate distance was kept at $55 \mathrm{~mm}$.

Grazing incidence $x$-ray diffraction (GXRD) in geometry at an angle of incidence of $0.75^{\circ}$ was utilized in order to study the structure of the films. The diffraction patterns have been recorded by using a Phillips X'pert Pro MRD diffractometer. The thickness of the films was measured with a profilometer. Dividing the film thickness by the deposition time, the deposition rate at various growth conditions was calculated. The film composition was determined by electron probe microanalysis (EPMA). In order to avoid postgrowth oxidation, a $20 \mathrm{~nm}$ thick Al layer was deposited on top of the films prior to the exposure to air for those samples that were prepared for EPMA measurements.

The optical properties of the films were investigated by employing variable angle spectroscopic ellipsometry. The spectra were recorded by a Woolam M-2000UI rotating compensator ellipsometer in the spectral range from 0.73 to $5.13 \mathrm{eV}$ at three angles of incidence, i.e., $50^{\circ}, 60^{\circ}$, and $70^{\circ}$. The measured data were fitted to a model consisting of a $\mathrm{ZrO}_{2}$ film on a glass substrate. The optical response of the $\mathrm{ZrO}_{2}$ film was parametrized by using the $O J L$ dispersion model proposed by O'Leary et al. ${ }^{15}$ and the optical constants, i.e., the refractive index $n$ and the extinction coeffi-

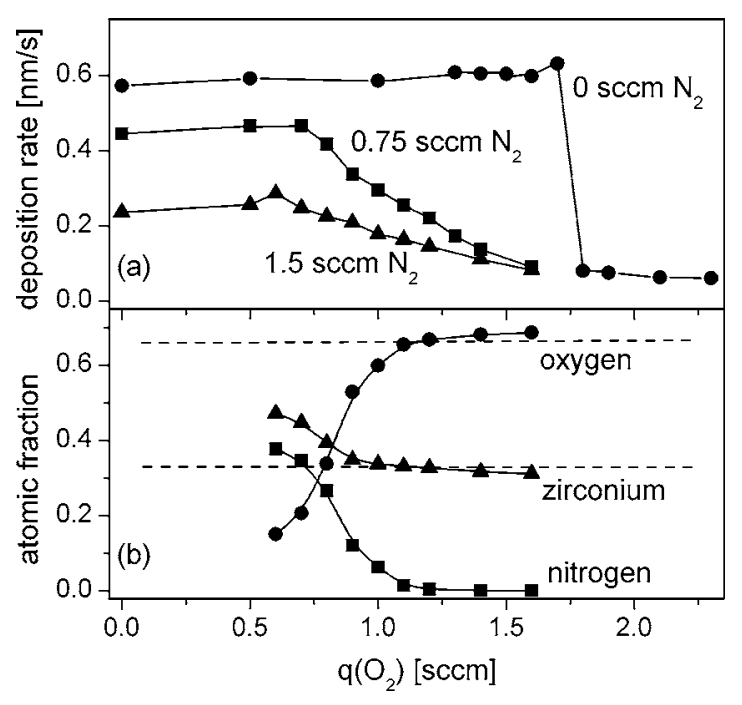

FIG. 1. (a) Deposition rate of reactively sputtered zirconium oxynitride with three different amounts of nitrogen addition. (b) Stoichiometry of the films deposited with $0.75 \mathrm{sccm}$ nitrogen addition. The broken lines present the nominal stoichiometry of $\mathrm{ZrO}_{2}$. These data are taken from Ref. 14

cient $k$, were determined to obtain the optical properties of the films over the investigated spectral range.

\section{RESULTS AND DISCUSSION}

\section{A. Process stability and arcing effect}

Figure 1(a) depicts the deposition rate as a function of increasing oxygen flow for three different amounts of nitrogen addition $(0,0.75$, and $1.5 \mathrm{sccm})$, and Fig. 1(b) shows the effect of the oxygen flow on the atomic fraction of zirconium, oxygen, and nitrogen for films sputtered with the addition of $0.75 \mathrm{sccm}$ nitrogen. These data are taken from Ref. 14. It can be clearly seen that the typical drop of the deposition rate from metallic to compound mode changes to a smooth transition upon increasing nitrogen addition. Furthermore, the nitrogen incorporation in the oxide film is very small for a sufficient oxygen flow. At $1.2 \mathrm{sccm}$ oxygen flow the nitrogen incorporation is less than $0.5 \%$. At the same time, the deposition rate is increased by a factor of approximately 3 compared to the film deposited with $1.8 \mathrm{sccm}$ oxygen flow and no nitrogen addition. The increased deposition rate is a result of the higher erosion rate of the nitride compared to the corresponding oxide since a significant fraction of the target surface is covered with a nitride instead of the oxide.

In addition to the improved deposition rate, the arcing during the deposition was reduced when nitrogen was added. Arcing leads in many cases to the appearance of macrodroplets on the film surface. During reactive sputtering of $\mathrm{ZrO}_{2}$ in the compound mode without nitrogen supply, we have observed a significant number of arcing events. On the other hand, the plasma discharge became very stable upon nitrogen addition. An origin of the arcing is the charge accumulation on the target surface when covered with an oxide leading to a dielectric breakthrough. Since the $\mathrm{ZrN}$ exhibits a metallic behavior, the higher electrical conductivity prevents the charge accumulation and results in less arcing events. 


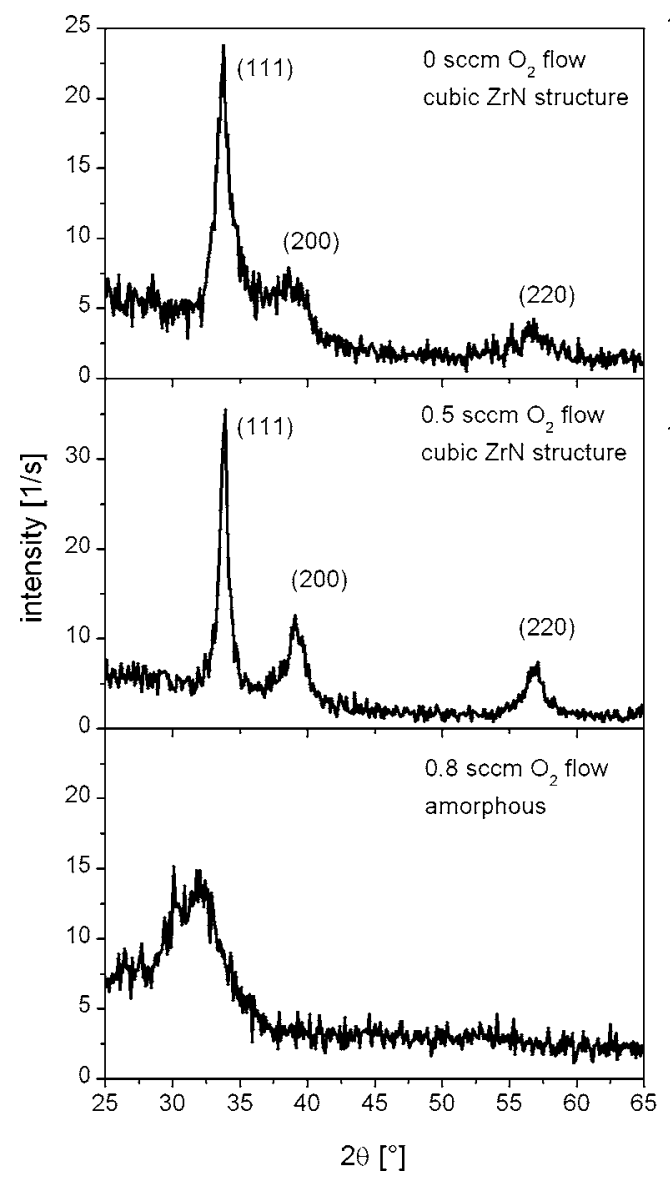

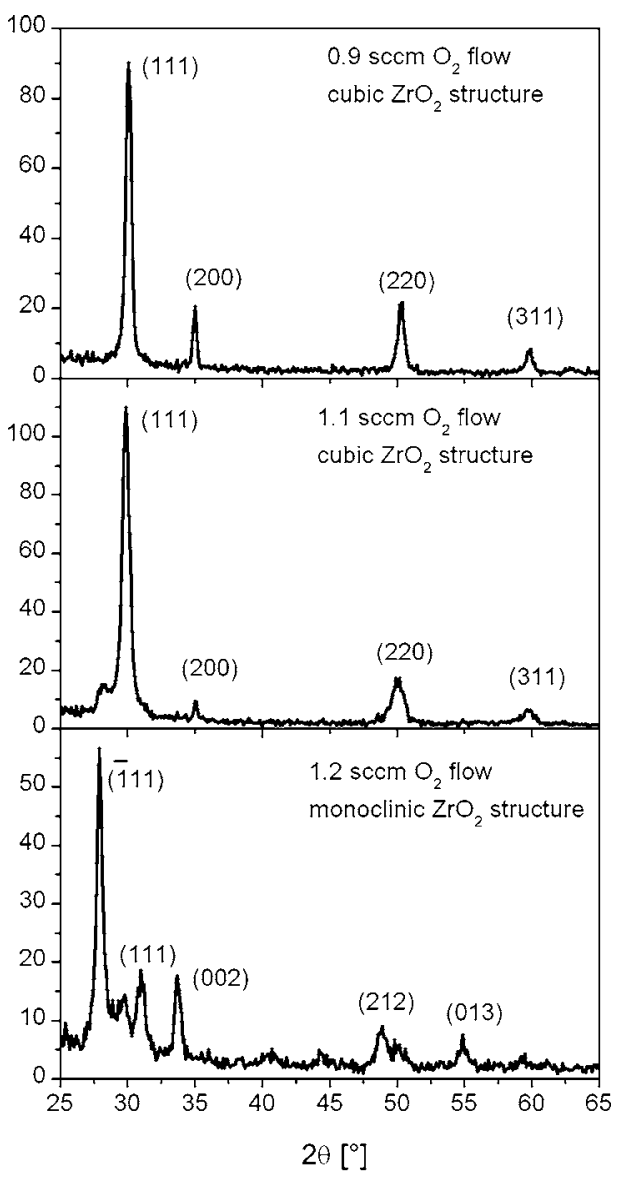

FIG. 2. Grazing angle XRD measurements for the $\mathrm{ZrO}_{x} \mathrm{~N}_{y}$ films deposited with $0.75 \mathrm{sccm}$ nitrogen addition and varying oxygen flow. The incidence angle chosen here is $0.75^{\circ}$. The structure switches from cubic $\mathrm{ZrN}$ structure at low oxygen flow to an amorphous state at $0.8 \mathrm{sccm} \mathrm{O}_{2}$ flow. Between 0.9 and $1.1 \mathrm{sccm}$, the cubic phase is deposited. From $1.2 \mathrm{sccm}$ oxygen flow, the monoclinic structure is obtained.

\section{B. Structure and phase composition}

$\mathrm{X}$-ray analysis was performed to correlate the structure formation with the working point. Figure 2 depicts the representative GXRD patterns of films sputtered at different $\mathrm{O}_{2}$ flows and $0.75 \mathrm{sccm} \mathrm{N}_{2}$ flow. With increasing oxygen flow, the structure changes from the cubic $\mathrm{ZrN}$ phase to an amorphous structure at $0.8 \mathrm{sccm}$. Between 0.9 and $1.1 \mathrm{sccm}$, an apparently cubic $\mathrm{ZrO}_{2}$ phase and above $1.2 \mathrm{sccm} \mathrm{O}_{2}$ flow the monoclinic phase are observed. Since the tetragonal and the cubic structures result in very similar XRD patterns, it is very difficult to distinguish both phases. For this reason, we have performed $\mathrm{x}$-ray scans with high resolution and a broad $2 \theta$ range (not shown here). The obtained pattern is in very good agreement with the cubic phase of the JCPDSInternational diffraction database. In contrast, the tetragonal phase is somewhat different; i.e., the measured peaks are shifted to slightly higher diffraction angles and no peak broadening due to the different lattice parameters of the tetragonal phase is apparent. Therefore, we conclude that the deposited phase is cubic. However, a small fraction of tetragonal grains cannot be ruled out.

Figure 3 depicts a summary of the different phases deposited at varying nitrogen and oxygen flows. The series sputtered without nitrogen shows the typical structure evolution of $\mathrm{ZrO}_{2}$ during reactive sputtering. Starting without any reactive gas, metallic $\mathrm{Zr}$ was obtained. The structure changes in the transition zone between the metallic and the compound modes to an amorphous phase. The final transition to the compound mode is characterized by a drop of the deposition rate due to the abrupt poisoning of the target surface with oxygen [see Fig. 1(a)]. In the compound mode, monoclinic films were obtained. The addition of nitrogen results in interesting significant features in the phase diagram, as discussed in the following.

(i) The structure evolution, i.e., the transitions between the phases, shifts to lower oxygen flows with increasing nitrogen flow. This is due to the decrease of the erosion rate in

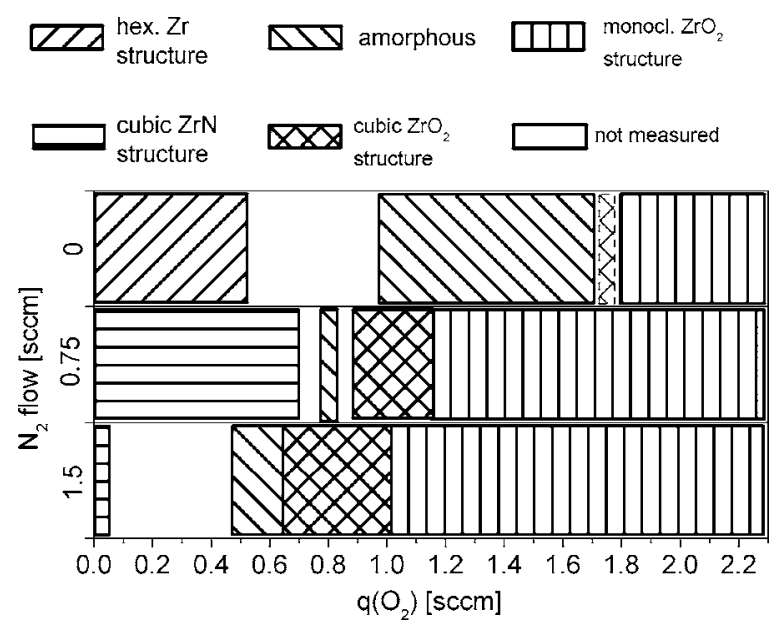

FIG. 3. Phase diagram of reactively sputtered $\mathrm{ZrO}_{x} \mathrm{~N}_{y}$ films for different oxygen and nitrogen flows. With increasing nitrogen addition, a stable process window to deposit the cubic phase is achieved. Furthermore, the transition to the monoclinic phase shifts to lower oxygen flow with increasing nitrogen addition. The dashed area at $0 \mathrm{sccm}$ nitrogen flow indicates that, in particular cases, a cubic oxide structure can be obtained. 
the metallic mode when nitrogen is added, as indicated by the smaller deposition rate [Fig. 1(a)] for small addition of oxygen. The decreased erosion rate is caused by a fraction of the target surface being covered with a nitride compound. This nitride compound exhibits a smaller erosion rate compared to the corresponding metal. In turn, less oxygen supply is needed in order to replace a metal-nitrogen bond by a more reactive metal-oxygen bond at the growing film surface leading to an oxide structure.

(ii) The transition from metallic to compound mode at $0 \mathrm{sccm} \mathrm{N}_{2}$ flow takes place at $1.8 \mathrm{sccm}$ oxygen flow as indicated by the drop of the deposition rate. In comparison, even a flow of $0.75 \mathrm{sccm}$ nitrogen is sufficient to deposit cubic nitride films. This discrepancy is caused by the different amount of reactive gas atoms in the compound. In order to obtain $\mathrm{ZrN}$ with nominal stoichiometry, only half of the amount of reactive gas molecules is required compared to the deposition of $\mathrm{ZrO}_{2}$. Furthermore, the nitride may exhibit an understoichiometric structure containing nitrogen vacancies. The addition of oxygen leads to reduced microstrain caused by filling the nitrogen vacancies with oxygen atoms. This is indicated by the decrease of the peak width of the cubic $\mathrm{ZrN}$ $x$-ray pattern when increasing the oxygen flow from 0 to $0.5 \mathrm{sccm}$ [Fig. 2].

(iii) The nitrogen addition results in a stable process window to deposit the cubic $\mathrm{ZrO}_{2}$ phase. It is important to point out here that the formation of the cubic phase is not a result of nitrogen incorporation in the growing film but is assigned to the tailored bombardment with energetic particles. In particular cases, the cubic phase could also be obtained without any nitrogen addition in the transition zone when the target coverage with oxygen is very small. This is indicated by the dashed area in Fig. 3 at $0 \mathrm{sccm}$ nitrogen flow. However, the process window for the deposition of the cubic phase is highly unstable in this case due to the nature of the unstable transition regime between the metallic and the compound mode and therefore hard to realize. This implies that the coverage of the target surface with oxygen and therefore the bombardment with highly energetic oxygen ions crucially affects the structure formation and the phase composition of $\mathrm{ZrO}_{2}$. The addition of nitrogen leads to a smaller and controllable fraction of oxygen compound on the target surface since a significant part is covered by the nitride. As a result, the bombardment with negatively charged oxygen ions and therefore the film structure can be tailored during reactive sputtering within a stable process window. The increase of the oxygen flow leads to an increased oxide fraction on the target surface and therefore to a more intense bombardment with negatively charged oxygen ions.

These findings are in good agreement with results obtained with IBAD by other groups. Without ion bombardment and without external substrate heating, amorphous $\mathrm{ZrO}_{2}$ films were deposited. The simultaneous bombardment with fast ions or substrate heating resulted in the formation of a cubic $\mathrm{ZrO}_{2}$ phase. ${ }^{1,16,17}$ This effect was attributed to thermal spikes leading to the nucleation of cubic grains. Since the surface energy of the cubic grains is smaller than that of monoclinic or tetragonal grains, these nuclei are expected to be stable at sufficiently small grain sizes. ${ }^{18}$ During

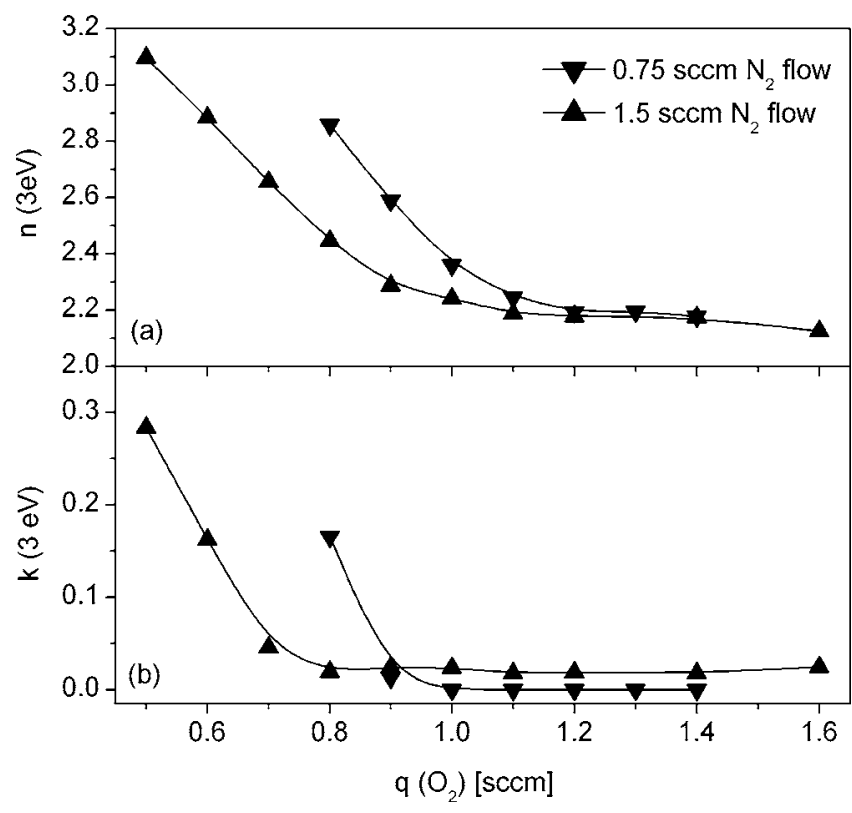

FIG. 4. Complex index of refraction at a photon energy of $3 \mathrm{eV}$ and varying nitrogen and oxygen flows. With increasing oxygen flow, the index of refraction decreases. At $1.5 \mathrm{sccm}$ nitrogen flow, a small absorption is left even at high oxygen flow.

reactive sputtering, the control of the target surface being covered by the oxide via the addition of nitrogen offers another opportunity to influence the bombardment and therefore the structure formation. Some of the negative oxygen ions might be neutralized due to scattering events as they traverse the plasma. However, due to the high kinetic energy, the scattering cross section is reduced. This leads to a mean free path of the order of several centimeters ${ }^{19}$ and therefore to high energetic input in the growing film.

\section{Optical properties}

An optical analysis was performed in order to correlate the nitrogen incorporation with the optical appearance. Figure 4 depicts (a) the refractive index and (b) the extinction coefficient at a photon energy of $3 \mathrm{eV}$ obtained by the spectroscopic ellipsometry for the series sputtered with 0.75 and $1.5 \mathrm{sccm} \mathrm{N}_{2}$ addition. The increase of the oxygen flow and therefore suppression of the nitrogen fraction in the films leads to a decreasing refractive index and extinction coefficient. At sufficient oxygen flow, both parameters saturate. The extinction is zero for the $0.75 \mathrm{sccm} \mathrm{N}_{2}$ series from 1 sccm $\mathrm{O}_{2}$ addition and above. This means that from this working point, fully transparent films can be obtained with only a little amount of nitrogen incorporation. In the case of the $1.5 \mathrm{sccm} \mathrm{N}_{2}$ series, there is still a small extinction of around $\sim 0.02$ left even at high $\mathrm{O}_{2}$ flow. This absorption in the visible range indicates that excessive nitrogen from the gas phase might be incorporated in the film.

These optical data show that transparent films with high deposition rate and variable film structure can be obtained by the addition of nitrogen during the growth process. In addition, the refractive index can be increased with lower oxygen flow. Similar results have also been published for reactively sputtered $\mathrm{ZrO}_{x} \mathrm{~N}_{y}$ in an oxygen-nitrogen atmosphere. ${ }^{2,20}$ It 
has been pointed out that the enhancement of the refractive index for small oxygen flow might be due to a shift of the band gap to lower values with increasing nitrogen incorporation.

\section{CONCLUSION}

In summary, our studies show that the addition of nitrogen during reactive sputtering of $\mathrm{ZrO}_{x}$ results in a controllable bombardment with negatively charged oxygen ions due to a stabilization of the hysteresis. Furthermore, the incorporation of nitrogen in the film is very small at sufficient oxygen flow. This is attributed to a lower reactivity of the nitride compared to the corresponding oxide. Nevertheless, the fraction of nitrogen on the target surface is higher since a metalnitrogen molecule at the target surface is more likely sputtered away before the nitrogen can be replaced by a more reactive oxygen atom. This leads to an enhanced deposition rate and less arcing. The controllable bombardment offers the opportunity to grow $\mathrm{ZrO}_{x} \mathrm{~N}_{y}$ with three different structural phases (amorphous, cubic, and monoclinic) without additional substrate heating. Finally, the refractive index can be increased with nitrogen addition.

\section{ACKNOWLEDGMENTS}

The authors would like to thank the Volkswagenstiftung (Germany) for financial support of this work.
${ }^{1}$ P. J. Martin, R. P. Netterfield, and W. G. Sainty, J. Appl. Phys. 55, 235 (1983).

${ }^{2}$ S. Venkataraj, O. Kappertz, H. Weis, R. Drese, R. Jayavel, and M. Wuttig, J. Appl. Phys. 92, 2461 (2002).

${ }^{3}$ S. Berg and T. Nyberg, Thin Solid Films 476, 215 (2005).

${ }^{4}$ K. Tominaga, S. Iwamura, Y. Shintani, and O. Tada, Jpn. J. Appl. Phys., Part 1 21, 688 (1982).

${ }^{5}$ K. Tominaga, T. Murayama, Y. Sato, and I. Mori, Thin Solid Films 343344, 81 (1999).

${ }^{6}$ K. Tominaga and T. Kikuma, J. Vac. Sci. Technol. A 19, 1582 (2001).

${ }^{7}$ M. Zeuner, H. Neumann, J. Zalman, and H. Biedermann, J. Appl. Phys. 83, 5083 (1998).

${ }^{8}$ R. Hippler, S. Wrehde, V. Stranak, O. Zhigalov, H. Steffen, M. Tichy, M. Quaas, and H. Wulff, Contrib. Plasma Phys. 45, 348 (2005).

${ }^{9}$ J. M. Ngaruiya, O. Kappertz, S. H. Mohamed, and M. Wuttig, Appl. Phys. Lett. 85, 748 (2004).

${ }^{10}$ S. Mraz and J. M. Schneider, J. Appl. Phys. 100, 023503 (2006).

${ }^{11}$ S. Mahieu and D. Depla, Appl. Phys. Lett. 90, 121117 (2006).

${ }^{12}$ D. Severin, O. Kappertz, T. Nyberg, S. Berg, and M. Wuttig, Thin Solid Films 515, 3554 (2006).

${ }^{13}$ O. Kappertz, R. Drese, and M. Wuttig, J. Vac. Sci. Technol. A 20, 2084 (2002).

${ }^{14}$ D. Severin, O. Kappertz, T. Kubart, T. Nyberg, S. Berg, A. Pflug, M. Siemers, and M. Wuttig, Appl. Phys. Lett. 88, 161504 (2006).

${ }^{15}$ S. K. O'Leary, S. R. Johnson, and P. K. Lim, J. Appl. Phys. 82, 3334 (1997).

${ }^{16}$ A. S. Kao and G. L. Gormanm, J. Appl. Phys. 67, 3826 (1990).

${ }^{17}$ R. Rujkorakarn and J. R. Sites, J. Vac. Sci. Technol. A 4, 568 (1986).

${ }^{18}$ F. Jones, J. Vac. Sci. Technol. A 6, 3088 (1988).

${ }^{19}$ J. M. E. Harper, J. J. Cuomo, R. J. Gambino, H. R. Kaufman, and R. S. Robinson, J. Vac. Sci. Technol. 15, 1597 (1978).

${ }^{20}$ S. Venkataraj, D. Severin, S. H. Mohamed, J. Ngaruiya, O. Kappertz, and M. Wuttig, Thin Solid Films 502, 228 (2006). 